\title{
La salute del paziente in dialisi e le nostre mani Il contributo infermieristico alla prevenzione delle infezioni associate alle organizzazioni sanitarie nei Centri Dialisi della AUSL di Rimini
}

\author{
Marina Sgreccia $^{1}$, Tatiana Fronzoni ${ }^{1}$, Elisa Ticchi ${ }^{1}$, Nicole Marcatelli ${ }^{2}$, Roberta Nicoletti ${ }^{3}$, \\ Barbara Zanchi ${ }^{3}$, Elisa Garattoni ${ }^{4}$, Grazia Antonella Tura ${ }^{2}$ \\ ${ }^{1}$ Centro Dialisi Ospedaliero, Ospedale Infermi, Rimini \\ ${ }^{2}$ Settore Rischio Infettivo, Azienda USL di Rimini, Rimini \\ ${ }^{3}$ Centro Dialisi Assistenza Limitata Ospedale Ceccarini, Riccione \\ ${ }^{4}$ Centro Dialisi Assistenza Limitata Ospedale Franchini, Santarcangelo
}

\begin{abstract}
The HEALTH OF DIALYSIS PATIENTS IS IN OUR HANDS
Abstract. Infections contracted during hemodialysis are an important aspect of the issue of healthcare-associated infections. The dialyzed patient has a very high risk of contracting infections that may be life threatening. Only strict application of proper nursing standards, in addition to surveillance and good patients' management, will allow lowering the incidence of infections. The project aims at improving guidelines adherence of hands hygiene, through the use of e-learning and training strategies at the workplace.
\end{abstract}

Key words: Hand hygiene, Hemodialysis, Guidelines implementation

Conflict of interest: None.

Financial support: None.

Ricevuto: 16 Giugno 2013; Accettato: 9 Settembre 2013

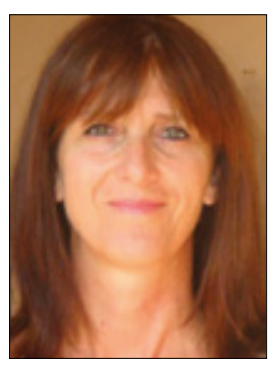

Marina Sgreccia

\section{Introduzione}

Le infezioni associate alle organizzazioni sanitarie (IOS) colpiscono ogni anno nel mondo centinaia di milioni di pazienti. Queste infezioni, che rappresentano un effetto indesiderato dell'assistenza, sono responsabili di aggravamento della patologia di base, prolungamento della degenza e disabilità a lungo termine (1). I pazienti emodializzati sono a elevato rischio infettivo, perché la pratica dialitica richiede la presenza di un accesso vascolare centrale per periodi prolungati. In un ambiente in cui molti pazienti vengono sottoposti contemporaneamente alla dialisi, si verificano numerosi casi di trasmissione "crociata" di agenti infettivi da paziente a paziente e da operatore a paziente, direttamente o indirettamente tramite dispositivi e apparecchiature e superfici contaminati nonché tramite le mani del personale (2-4). Inoltre, i pazienti dializzati sono immunodepressi, quindi maggiormente suscettibili a infezioni, per cui vengono frequentemente ricoverati e sottoposti a manovre invasive; ciò li espone ancora di più al rischio di $\operatorname{IOS}(4,5)$ Le infezioni batteriche, correlate a catetere venoso (CR-BSI), rappresentano le più frequenti complicanze infettive della pratica emodialitica e la maggiore causa di morbilità e mortalità per i pazienti stessi (3). Il tasso annuale di letalità tra di loro è del 23\%; le infezioni rappresentano la terza causa di decesso, essendo responsabili del 13\% delle morti; la sepsi (causa del $10.9 \%$ del totale dei decessi) è la più comune causa infettiva di mortalità $(6,7)$.

La letteratura internazionale ci offre valutazioni sull'efficacia dell'implementazione delle Linee Guida sull'igiene delle mani, prendendo in considerazione proprio il contesto dialitico e sottolineando, peraltro, che uno dei più comuni batteri che possono infettare il paziente dializzato è lo stafilococco MRSA e che il settore di Emodialisi possiede un elevato rischio intrinseco di trasmissione crociata (4). L'applicazione delle Linee Guida sull'igiene delle mani e la corretta tecnica asettica nelle manovre di connessione/distacco al rene artificiale sono fondamentali per minimizzare il rischio di infezione nel paziente dializzato: esiste, però, un "gap" tra le conoscenze e la pratica clinica (4). Tra le varie strategie multimodali per l'implementazione (1), viene data particolare rilevanza 
alla formazione del personale $(8,9)$. Nelle Linee Guida OMS sull'igiene delle mani nel capitolo dedicato al contesto emodialitico è chiaramente esplicitato l'elevato rischio di trasmissione di germi tra un paziente per l'invasività delle manovre di assistenza (10).

L'utilizzo di un accesso vascolare, ben funzionante, è indispensabile per effettuare una dialisi adeguata (11). La gestione degli accessi vascolari in emodialisi (fistola arterovenosa, protesi e cateteri venosi centrali) è una competenza infermieristica ormai diffusa e confermata da studi nazionali e internazionali dell'Associazione Europea Infermieri Dialisi e Trapianto (E.D.T.N.A.) (12). Questo progetto si è proposto l'obiettivo di implementare le Linee Guida sull'igiene delle mani tramite un processo formativo in modalità e-learning e sul campo.

\section{Obiettivi dello studio}

Un obiettivo tecnico principale: tendere al 100\% di aderenza alle Linee Guida sull'igiene delle mani per aumentare la sicurezza del paziente emodializzato.

Un obiettivo metodologico: insegnare agli infermieri ad accogliere i cambiamenti nel proprio ambito professionale.

Un obiettivo formativo: il valore aggiunto dell'insegnamento che si coglie nel lavorare basandosi sull'evidenza scientifica.

\section{Materiali e Metodi}

Contesto. Nell'Azienda USL di Rimini sono attivi tre Centri Dialisi: un Centro Ospedaliero (presso l'Ospedale di Rimini) e due Centri di Assistenza Limitata (presso l'Ospedale di Riccione e l'Ospedale di Santarcangelo). Questi tre Centri forniscono un totale di 57 posti letto dialisi per pazienti con insufficienza renale cronica terminale attivi su 12 ore. I pazienti afferenti ai tre Centri sono circa 200/anno; il numero medio di trattamenti annui è pari a 31000; nel rispetto dei requisiti di accreditamento della Regione Emilia Romagna (13), che prevedono l'assegnazione di un numero massimo di tre pazienti per ogni infermiere, l'organizzazione del lavoro infermieristico scelta nei tre Centri è quella per settori: a ognuno di essi è assegnato un infermiere che provvede alla gestione della seduta dialitica di un numero massimo di tre pazienti.

Sono attivi presso i tre Centri Dialisi da alcuni anni due sistemi di sorveglianza di eventi avversi (anche infettivi) a carico degli accessi vascolari: il primo interessa la popolazione dei pazienti portatori di catetere venoso centrale (14) e il secondo riguarda i pazienti arruolati per la metodica di puntura della FAV denominata "a occhiello". A seguito della campagna aziendale sull'implementazione delle Linee Guida sull'igiene delle mani, i Centri Dialisi aziendali hanno aderito dal 2011 al progetto di miglioramento.

Protocollo dello studio. È stato utilizzato il protocollo italiano del progetto dell'OMS “Sfida mondiale per la sicurezza del paziente - Cure pulite sono cure più sicure" (Global Patient Safety Challenge - Clean Care is Safer Care) (15), che prevede le seguenti fasi operative:

- preparazione della struttura e analisi strutturale pre-post

- acquisizione di soluzioni alcoliche per l'igiene delle mani

- formazione e addestramento degli osservatori e del personale di reparto

- distribuzione di poster, opuscoli e reminder

- osservazione della compliance prima e dopo (almeno 200 osservazioni)

- elaborazione e restituzione dei dati all'equipe

Popolazione dello studio. Oggetto di questo studio è stato rappresentato dagli infermieri, in quanto maggiormente coinvolti nell'assistenza diretta (30 per il Centro di Rimini, 12 per quello di Riccione, 10 per quello di Santarcangelo).

\section{Strumenti}

A seguito di due corsi di formazione residenziale organizzati dall'U.O. Nefrologia e Dialisi dell'Azienda USL di Rimini riguardanti la prevenzione delle infezioni associate alle organizzazioni sanitarie, è stata valutata dai Coordinatori Infermieristici dei Servizi di Dialisi e dai Responsabili Infermieristici del Settore Rischio Infettivo Aziendale la possibilità di valutare direttamente la percentuale di aderenza alle Linee Guida sull'igiene delle mani; fino ad allora, infatti, l'unico sistema di valutazione indiretto era rappresentato dal consumo di gel alcolico. Dal momento che, presso ogni postazione infermieristica di lavoro, sono presenti i contenitori del gel alcolico, vengono affissi i poster informativi forniti dalla Regione (9) riguardanti il corretto utilizzo del prodotto e i cinque momenti fondamentali dell'igiene delle mani. Attraverso la collaborazione con il Settore Rischio Infettivo sono state formate tre infermiere del servizio di Dialisi, affinché potessero acquisire la competenza necessaria per compiere l'osservazione e la rilevazione dei dati con la scheda messa a disposizione sul sito della Regione (9). Una prima osservazione e rilevazione dati è stata svolta tra Ottobre e Novembre 2011 nel Centro Dialisi di Rimini, così da trarre i primi dati percentuali; a questo punto, è stata offerta agli infermieri una formazione specifica in modalità e-learning sull'igiene delle mani, disponibile sulla piattaforma della formazione a distanza (FAD) di Area Vasta Romagna (http://www.e-romagna.it/). La durata del corso è stata di 9 ore; la difficoltà è stata definita media; l'obiettivo dichiarato è stato il seguente: "implementare la pratica del lavaggio delle mani nei vari ambienti di cura, riconoscendo il tipo di lavaggio e il materiale da usare nelle varie situazioni dell'attività professionale e usando la buona pratica assistenziale"; i moduli che lo componevano erano i seguenti:

- prologo sulla storia dell'igiene delle mani

- anatomia e fisiologia delle mani

- flora microbica cutanea

- igiene delle mani e infezioni ospedaliere

- prodotti per l'igiene delle mani

- tecniche per il lavaggio delle mani

- raccomandazioni delle Linee Guida

- strategie di miglioramento dell'aderenza alla pratica dell'igiene delle mani

Nel primo e nell'ultimo trimestre dell'anno 2012 le osservazioni sono state ripetute e i dati sono stati confrontati con quelli raccolti dopo la formazione specifica. La medesima metodica è stata utilizzata nel Centro di Assistenza Limitata di Riccione: le osservazioni sono state compiute nel primo trimestre del 2012; è stata, poi, offerta la formazione e le osservazioni sono state ripetute nel secondo trimestre. Infine, nel primo trimestre 
del 2013, ha aderito al progetto anche il Centro di Assistenza Limitata di Santarcangelo: il personale infermieristico ha partecipato alla formazione a distanza, ma, soprattutto, ha avuto modo di conoscere e di analizzare al proprio interno i dati degli altri due Centri Dialisi.

\section{Risultati}

Nella prima fase di osservazione (dal 22/09/2011 al 08/11/2011), nel Centro Dialisi di Rimini, è stato compiuto un totale di 221 osservazioni, che hanno portato ai risultati riportati nella Tabella I.

TABELLA I - DATI DEL CENTRO DIALISI DI RIMINI, 2011

\begin{tabular}{|l|c|}
\hline Centro Dialisi di Rimini & Prima fase \\
\hline Aderenza generale & $42.53 \%$ \\
\hline Prima del contatto con il paziente & $30.43 \%$ \\
\hline Prima di una manovra asettica & $31.91 \%$ \\
\hline Dopo contatto/rischio con fluido corporeo & $32.14 \%$ \\
\hline Dopo un contatto con il paziente & $50 \%$ \\
\hline Dopo il contatto con l'ambiente vicino al paziente & $44.18 \%$ \\
\hline
\end{tabular}

Nella seconda fase di osservazione (dal 13/02/2012 al $02 / 03 / 2012$ ) è stato compiuto un totale di 413 osservazioni, che hanno prodotto i primi valori percentuali in crescita; nella terza fase di osservazione, svoltasi dal 20/08/2012 al 01/09/2012, è stato compiuto un totale di 407 osservazioni: i dati raccolti dimostrano un ulteriore miglioramento generale, come descritto nella Tabella II.

TABELLA II - DATI DEL CENTRO DIALISI DI RIMINI, 2012

\begin{tabular}{|l|c|c|}
\hline Centro Dialisi di Rimini & $\begin{array}{c}\text { Seconda } \\
\text { fase }\end{array}$ & $\begin{array}{c}\text { Terza } \\
\text { fase }\end{array}$ \\
\hline Aderenza generale & $64.40 \%$ & $69.36 \%$ \\
\hline Prima del contatto con il paziente & $60 \%$ & $64.19 \%$ \\
\hline Prima di una manovra asettica & $53.3 \%$ & $64.77 \%$ \\
\hline Dopo contatto/rischio con fluido corporeo & $62.25 \%$ & $33.42 \%$ \\
\hline Dopo un contatto con il paziente & $76.92 \%$ & $71.13 \%$ \\
\hline Dopo il contatto con l'ambiente vicino al paziente & 54.83 & $52.38 \%$ \\
\hline
\end{tabular}

Nel Centro di Assistenza Limitata di Riccione sono state svolte due fasi di osservazione: la prima dal 23/01/2012 al 23/02/2012, per un totale di 430 osservazioni, e la seconda dal 31/08/2012 al 27/09/2012, per un totale di 444 osservazioni. I risultati, sostanzialmente stabili, sono descritti nella Tabella III.

TABELLA III - DATI DEL CENTRO DIALISI DI RICCIONE, 2012

\begin{tabular}{|l|c|c|}
\hline CAL Riccione & Prima fase & $\begin{array}{c}\text { Seconda } \\
\text { fase }\end{array}$ \\
\hline Aderenza generale & $79.30 \%$ & $74.90 \%$ \\
\hline Prima del contatto con il paziente & $74.35 \%$ & $79.45 \%$ \\
\hline Prima di una manovra asettica & $87.14 \%$ & $81.11 \%$ \\
\hline Dopo contatto/rischio con fluido corporeo & $80.11 \%$ & $70 \%$ \\
\hline Dopo un contatto con il paziente & $63.79 \%$ & $66.66 \%$ \\
\hline $\begin{array}{l}\text { Dopo il contatto con l'ambiente vicino al } \\
\text { paziente }\end{array}$ & $80 \%$ & $80 \%$ \\
\hline
\end{tabular}

Infine, anche il Centro di Assistenza Limitata di Santarcangelo ha partecipato al progetto; sono state compiute 667 osservazioni (dal 7/01/2013 al 19/01/2013) e i risultati si sono mostrati subito più vicini ai primi dati del Centro di Riccione, come indicato nella Tabella IV.

TABELLA IV - DATI DEL CENTRO DI SANTARCANGELO, 2013

\begin{tabular}{|l|c|}
\hline Aderenza generale & $71.66 \%$ \\
\hline Prima del contatto con il paziente & $77.18 \%$ \\
\hline Prima di una manovra asettica & $60 \%$ \\
\hline Dopo contatto/rischio con fluido corporeo & $69.50 \%$ \\
\hline Dopo un contatto con il paziente & $73 \%$ \\
\hline Dopo il contatto con l'ambiente vicino al paziente & $72 \%$ \\
\hline
\end{tabular}

L'adesione degli infermieri alla formazione in modalità $e$-learning è stata pari al $75 \%$. Tutti i partecipanti hanno superato la prova finale e nessuno di essi aveva mai svolto un corso di formazione specifico dedicato esclusivamente all'argomento igiene delle mani. Questo tipo di modalità ha velocizzato $\mathrm{i}$ tempi di formazione di gruppo.

\section{Discussione}

Come si può evincere dai dati, in seguito alla formazione specifica, all'utilizzo di poster e alla presenza stessa degli osservatori interni al reparto, l'aderenza generale alle Linee Guida sull'igiene delle mani è aumentata del $26.83 \%$ nel Centro Dialisi di Rimini; in particolare, è positiva la costante di crescita della percentuale specifica relativa a "prima di una manovra asettica" e "prima del contatto con il paziente"; l'ambiente della dialisi si caratterizza, infatti, proprio per l'alta frequenza di manovre che richiedono l'asepsi, e la crescita di queste colonne nel grafico sta a validare la "tenuta" del cambiamento nel tempo. Il Centro di Assistenza Limitata di Riccione è partito da subito con dei dati sicuramente migliori ed essi tendono a mantenersi stabili nel tempo anche grazie al minor numero di pazienti presenti per turno e al numero inferiore di trattamenti urgenti. Anche il Centro di Santarcangelo ha dimostrato dati di partenza di gran lunga migliori rispetto a Rimini e più simili a quelli di Riccione. I dati elaborati e confrontati nel tempo sono stati esposti a tutta l'equipe medico-infermieristica dell'U.O. nel corso di due incontri denominati "Condivisione degli obiettivi assistenziali", durante i quali non solo è stata manifestata la soddisfazione per i risultati raggiunti, ma sono state anche esplicitate le maggiori difficoltà incontrate sia dagli osservatori che dagli operatori osservati.

Le maggiori difficoltà riportate dagli osservatori sono state di natura relazionale con il resto del gruppo, che non era abituato a sentirsi valutato; per i componenti del gruppo la parte più difficile è stata far accettare al paziente il tempo per l'igiene delle mani e per il cambio dei guanti tra una manovra "sporca" e una "pulita" o tra un paziente e l'altro.

Va detto, però, che la dialettica nel gruppo ha, comunque, "aperto le menti" e che i pazienti più giovani hanno accettato bene questo cambiamento. 
Uno degli ostacoli più grandi da superare soprattutto all'inizio (e ancora presente, in minima parte) è l'utilizzo inappropriato dei guanti monouso, indossati anche dove non era necessario e/o utilizzati come sostituti dell'igiene delle mani.

\section{Conclusioni}

L'esperienza fatta nell'ultimo anno porta a considerare come la lotta alle infezioni correlate all'assistenza sanitaria debba essere compiuta contemporaneamente su più fronti. La formazione rappresenta una delle più efficaci strategie per il miglioramento. Per il futuro sarà necessario ripetere le osservazioni e confrontare i progressi compiuti: l'indicazione del protocollo dell'OMS prevede che le osservazioni vengano effettuate trimestralmente. All'interno di ogni servizio ci si deve confrontare con l'effettiva applicabilità di questo requisito, poiché non è sempre possibile sottrarre all'assistenza diretta un osservatore dedicato, ma, quando questo è possibile, l'esperienza arricchisce, rende tutti i componenti del gruppo più consapevoli delle proprie azioni e offre un segnale forte anche all'utenza il cui benessere rimane al centro dell'agire infermieristico.

\section{Riassunto}

Le infezioni acquisite in dialisi sono un importante aspetto del più grande capitolo riguardante le infezioni correlate all'assistenza. I pazienti in emodialisi sono una popolazione a elevato rischio di infezione. La rigorosa applicazione delle Linee Guida sull'igiene delle mani può contribuire a ridurre l'incidenza delle infezioni. Il progetto di miglioramento mira ad aumentare l'aderenza alle Linee Guida sull'igiene delle mani anche attraverso l'utilizzo di strategie formative in modalità e-learning e sul campo.

Parole chiave: Igiene delle mani, Emodialisi, Implementazione delle Linee Guida

Dichiarazione di conflitto di interessi: Gli Autori dichiarano di non avere conflitto di interessi.

Contributi economici agli Autori: Gli Autori dichiarano di non aver ricevuto sponsorizzazioni economiche per la preparazione dell'articolo.

\section{Indirizzo degli Autori: \\ Dr.ssa Marina Sgreccia \\ Via Flaminia 199 \\ 47924 Rimini \\ msgreccia@auslrn.net}

\section{Bibliografia}

1. Zotti C, Moro ML (a cura di). Compendio delle principali misure per la prevenzione e il controllo delle infezioni correlate all'assistenza. Progetto INF-OSS 2010.

2. Linee Guida OMS sull'igiene delle mani nell'assistenza sanitaria. First Global Patient Safety Challenge - Clean Care is Safer Care 2009.

3. Infection Control Requirements for Dialysis Facilities and Clarification Regarding Guidance on Parenteral Medication Vials. MMWR Weekly August 15, 2008; 57 (32); 875-6.

4. Implementing change in Hand Hygiene and asepsis in Haemodialysis nursing care, disponibile sul sito http://publications.theseus.fi/bitstream/handle/10024/22160/IMPLEMENTING\%20 CHANGE\%20IN\%20HAND.pdf? sequence $=1$.

5. Movilli E. Effetto degli stimoli infiammatori sull'outcome clinico nei pazienti in dialisi. [Effect of inflammation on clinical outcomes in dialysis patients]. G Ital Nefrol 2006; 23 (3): 267-72.

6. Registro Regionale Emiliano Romagnolo di Dialisi e Trapianto; report anno 2010.

7. Gattuso G, Tomasoni D, Chiarelli C, Stradoni R, Scalzini A. Prevenzione e Gestione delle Infezioni da Germi Antibioticoresistenti nei pazienti emodializzati. [Prevention and management of antibiotic-resistant bacterial infections in patients on hemodialysis]. G Ital Nefrol 2010; 27 (Suppl. 52): S66-72.

8. Mongardi M, Moro ML, Callea E. Memo 7 Igiene delle Mani. Re- gione Emilia Romagna, Agenzia Saniatria e Sociale Regionale.

9. Arenas MD, Sánchez-Payá J, Barril G, et al. A multicentric survey of the practice of hand hygiene in haemodialysis units: factors affecting compliance. Nephrol Dial Transplant 2005; 20: 1164-71.

10. WHO. Hand Hygiene in Outpatient and Home-based Care and Long-term Care Facilities. A Guide to the Application of the WHO Multimodal Hand Hygiene Improvement Strategy and the "My Five Moments for Hand Hygiene" Approach.

11. Vascular Access Work Group. Clinical practice guidelines for vascular access. Am J Kidney Dis 2006; 48 (Suppl. 1): S248-73.

12. Pegoraro M. La gestione dell'Accesso Vascolare nei pazienti in dialisi: il contributo dello Studio DOPPS. Giornale di Tecniche Nefrologiche \& Dialitiche 2010: 22 (1): 34-5.

13. Requisiti specifici per l'accreditamento dei Centri Dialisi della Regione Emilia Romagna, disponibile sul sito http://assr.regione.emilia-romagna.it/it/servizi/pubblicazioni/legislazione-e-attiamministrativi/requisiti-accreditamento/requisiti-specifici/req-snefrologia-dialisi/?searchterm=emodialisi.

14. GIMPIOS Giornale Italiano Multidisciplinare per la Prevenzione delle Infezioni nelle Organizzazioni Sanitarie N. 1, Gennaio-Marzo 2011. Abstract delle comunicazioni e dei poster presentati al $4^{\circ}$ Congresso Nazionale Simpios, 10-12 Maggio 2010, Poster $\mathrm{N}^{\circ}$ A4.

15. Protocollo Italiano del progetto OMS. Sfida mondiale per la sicurezza del paziente - Cure Pulite sono cure più sicure, disponibile sul sito http://www.ccm-network.it/documenti_Ccm/prg_area1/ Inf_Oss/Lg_internaz/LG_Oms_05-06.pdf. 